\title{
Benefits of the Combined Use of NSAID and Early Exercise on Tendon Healing in a Murine Model
}

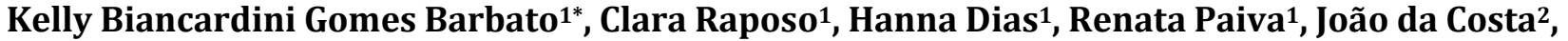 Libardo Rodriguez², Guilherme de Almeida², Oscar Rocha-Barbosa ${ }^{3}$, Liszt Palmeira de Oliveira², Jorge Carvalho 4}

${ }^{1}$ School of Medicine, State University of Rio de Janeiro, Rio de Janeiro, Brazil

${ }^{2}$ Department of Surgical Specialties, School of Medicine, State University of Rio de Janeiro, Rio de Janeiro, Brazil

${ }^{3}$ Department of Zoology, Roberto Alcantara Gomes Institute of Biology, State University of Rio de Janeiro, Rio de Janeiro, Brazil

${ }^{4}$ Department of Histology and Embriology, Roberto Alcantara Gomes Institute of Biology, State University of Rio de Janeiro,

Rio de Janeiro, Brazil

Email: *kelbiancardini@gmail.com, lisztpalmeira@yahoo.com.br

How to cite this paper: Barbato, K.B.G., Raposo, C., Dias, H., Paiva, R., da Costa, J., Rodriguez, L., de Almeida, G., Rocha-Barbosa, O., de Oliveira, L.P. and Carvalho, J. (2018) Benefits of the Combined Use of NSAID and Early Exercise on Tendon Healing in a Murine Model. Open Journal of Animal Sciences, 8, 357-369. https://doi.org/10.4236/ojas.2018.84027

Received: June 25, 2018

Accepted: August 31, 2018

Published: September 3, 2018

Copyright (c) 2018 by authors and Scientific Research Publishing Inc. This work is licensed under the Creative Commons Attribution International License (CC BY 4.0).

http://creativecommons.org/licenses/by/4.0/

\section{(c) (i) Open Access}

\begin{abstract}
Achilles tendon rupture is one of the most frequent tendon injuries. The aim of this experimental study was to evaluate the effect of a non-steroidal anti-inflammatory drug (NSAID) and of aerobic exercise, in an isolated fashion and combined, on cell proliferation and on the biomechanical aspects of the Achilles tendon during healing process after a complete tenotomy in 156 male Wistar rats. Half of the animals received an intra-muscular injection of tenoxicam for 7 days and exercise was initiated on the 8th day for half the animals of each group. Animals were sacrificed at 1,2, 4 and 8 weeks after the tenotomy and cell proliferation was evaluated by immunohistochemistry for PCNA, biomechanical evaluation was performed with ultimate load and gait cycle analysis was also carried out. Animals were divided into four treatment groups: A) no NSAID and no exercise (control group); B) NSAID plus exercise; C) no NSAID, with exercise; D) NSAID and no exercise. We used the test of two-way analysis of variance with equal cells number, Kruskal-Wallis test and also, Bonferroni method, in the R Project program 3.5.0. The highest intensity of PCNA immunostaining was found at 2 weeks in all groups except for group A (control) that had the highest intensity at 1 week. Animals submitted to exercise had significantly higher $(P=0.02)$ ultimate loads when compared to animals that were not submitted to exercise at 8 weeks after injury. The animals that received NSAID presented with a more stable gait cycle than the animals that did not receive NSAID. Aerobic exercise, initiated
\end{abstract}


early after a complete Achilles tendon tenotomy, was beneficial to the biomechanical aspects of the tendon that occur during tendon regeneration and the combined use of NSAID improved the gait's characteristics which could be protective against re-ruptures.

\section{Keywords}

Achilles Tendon Rupture, Experimental Study, Non-Steroidal

Anti-Inflammatory Drug, Aerobic Exercise, PCNA, Biomechanics

\section{Introduction}

Achilles tendon is the largest and strongest tendon of the lower limb but it is also the most frequently injured corresponding to $20 \%$ of tendon injuries [1]. Slow recovery, late return to work, high re-rupture rate and other complications characterize this tendon's rupture [2] [3]. In spite of this, treatment of this injury [4] [5] [6] and post injury mobilization [7] [8] [9] are still controversial.

Ciclooxygenases are enzymes that form prostaglandins, important inflammatory mediators [10]. Nonsteroidal anti-inflammatory drugs (NSAID), that inhibit ciclooxygenases, are commonly used during the first week following musculoskeletal traumas in order to diminish pain and the inflammatory response [11]. Nonetheless, concern exists regarding their possible adverse effects with the tendon's regeneration process. Many have shown negative effects on the proliferative phase of the tissue regeneration with inhibition of DNA synthesis, cell migration and tendon cell proliferation [12] but positive effects on the remodeling phase [13].

Although most studies have shown the benefits of exercise on tendon regeneration and functional recovery [14] [15], controversy still exists concerning its effect on the tendon's biomechanical properties. When ultimate load is taken into account, some have shown positive effects [16], negative effects [17] and no effect [18]. In addition to this, the effect of exercise combined to the use of NSAID has not been fully investigated. Christensen et al. [19] have reported a decrease in protein synthesis induced by exercise because of the combined use of NSAID in human patellar tendon but the same authors question if the use of the drug would be beneficial in the reduction of the collagen fibers degradation also associated with the acute inflammatory phase.

Our hypothesis is that exercises combined with NSAID tenoxicam can lead to a faster recovery of the affected area. Therefore, the aim of of this study is to evaluate the effects of exercise and the NSAID tenoxicam, in an isolated fashion and in combination, on cell proliferation and biomechanical aspects after a complete tenotomy of the Achilles tendon in a rat model.

\section{Methods}

The Animal Research and Care Committee at our University approved all experimental protocols for this study. 156 male Wistar rats aged 3 months with an 
average weight of $300 \mathrm{~g}$ were used for this experiment. All animals were allowed access to food (standard diet) and water ad libitum. Twelve-hour light/dark cycles were maintained, with temperatures at $21^{\circ} \mathrm{C} \pm 2^{\circ} \mathrm{C}$. The animals were kept in regular cages and allowed complete freedom to move in the cages.

Animals were randomly divided into 4 groups: group A: a control group with 42 animals (received no NSAID and no exercise); group B with 36 rats (received NSAID and exercise); group C with 36 rats (no NSAID, with exercise) and group $\mathrm{D}$ with 42 rats (received NSAID but no exercise).

\subsection{The Injury}

All animals were submitted to a tenotomy of the right Achilles tendon. For this, animals were anesthetized with an intraperitoneal injection of 30 to $50 \mathrm{mg} / \mathrm{kg}$ of thiopental (Thiopentax, Cristalia, Brazil). After the hind limb was disinfected, a posterior longitudinal incision of approximately $5 \mathrm{~mm}$ was made proximal to the calcaneus' posterior tuberosity and the plantaris tendon was identified and preserved. After this, a complete transverse incision of the Achilles tendon was made $7 \mathrm{~mm}$ proximally to the calcaneus tuberosity. The skin was sutured with non-absorbable sutures and no post-op immobilization was used. This improved experimental technique for complete Achilles tenotomy in rats, with plantaris tendon sparing, performed under light microscopy has been described before [20].

\subsection{Non-Steroidal Anti-Inflammatory Drug}

Animals from groups B and D received an intramuscular injection of tenoxicam (Eurofarma, Brazil). Animals received one dose $(10 \mathrm{mg} / \mathrm{kg})$ a day for 7 days initiated immediately before the injury. Dosage was based upon previous work by Giordano et al. [21].

Animals that did not receive NSAID (groups A and C) received an intramuscular injection of saline solution, once a day for 7 days.

\subsection{Exercise Protocol}

Beginning at the 8th day after injury animals from groups $\mathrm{B}$ and $\mathrm{C}$ were submitted to a running protocol on a treadmill (Insight, Ribeirão Preto, Brazil): they ran 5 times a week with a progressive increase in time and speed until they ran for 30 minutes at $14 \mathrm{~m} / \mathrm{min}$ for 1,3 or 7 weeks. This protocol is considered low intensity exercise [22]. Animals from groups A and D that were not submitted to the exercise protocol were placed on the treadmill for 30 minutes but the machine was left off.

\subsection{Outcomes}

The animals were sacrificed by sodium thiopental overdose at 1, 2, 4 and 8 weeks after the tenotomy. 


\subsubsection{Histology}

Histology was performed 1, 2, 4 and 8 weeks after tenotomy in groups A and C (6 animals in each group for each time point) and after 2, 4 and 8 weeks in groups $\mathrm{B}$ and $\mathrm{D}$ ( 6 animals in each group for each time point).

The right Achilles tendon was dissected and fixed in 10\% formaldehyde, embedded in paraffin and $5 \mu \mathrm{m}$ cuts were made. Immunohistochemistry for proliferating cell nuclear antigen (PCNA, Santa Cruz Biotechnology, OR, USA) was performed.

\subsubsection{Biomechanics}

Biomechanical evaluation was performed 2, 4 and 8 weeks after tenotomy (6 animals per group per time point) on a universal traction machine type E 200. For this, the distal end of the triceps surae is fixed in the superior clamp and the animal's paw is fixed in the inferior clamp. The ultimate loads were measured in Newton.

\subsubsection{Gait Analysis}

Two animals from group $\mathrm{B}$ and two animals from group $\mathrm{D}$ were randomly chosen each week and their gait, on the treadmill, was recorded with a Casio digital camera (Casio Exilim F1) from week 1 to week 7.

30 frames per second were obtained with the camera and, for each animal, 10 seconds of film was evaluated 3 times, at different time points, by the same observer. The parameters observed were: time of stance phase, time of swing phase, total cycle time (stance phase + swing phase) and pace frequency for the injured hind limb.

\subsubsection{Statistical Analysis}

The statistical analysis was performed in free software R ( $R$ Project, version 3.5.0). In data analysis, we used the test of analysis of variance (two-way analysis of variance with equal cells number), the nonparametric Kruskal-Wallis test and also, Bonferroni method. Graphical models were used to supplement the analysis. Statistical significance was set at 0.05 .

\section{Results}

\subsection{Histology}

At one week after tenotomy the intensity of PCNA immunostaining was low in group D (NSAID group) and high in group A (non NSAID group) (Figure 1).

At two weeks after tenotomy, weak immunostaining was found in group A and high immunostaining was found in groups B, C and D that received NSAID and/or exercise. In these 3 groups, the highest marking was found at the musculotendinous junction when compared to the tendon's mid-substance (Figure 2).

At four weeks after tenotomy, the intensity of PCNA immunostaining was low in all groups. In group A, the highest marking was found at the musculotendinous 


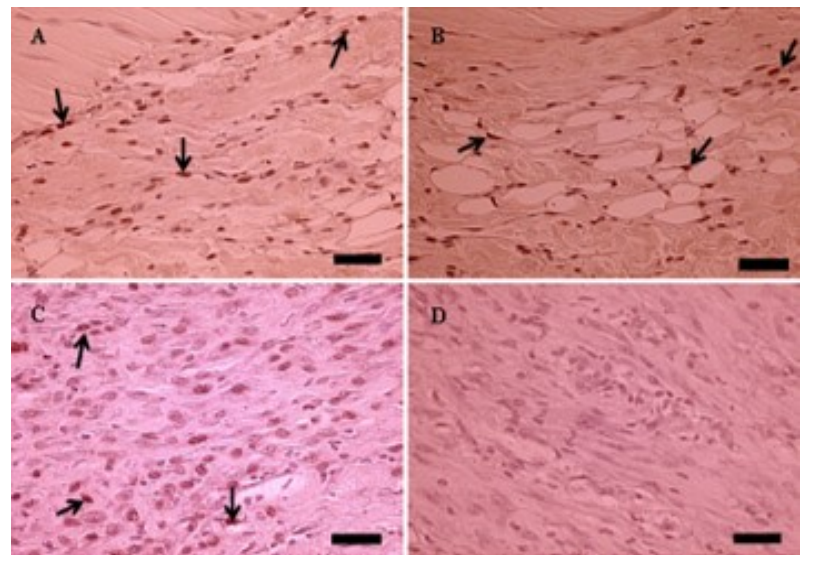

Figure 1. Histology of the longitudinal section of the Achilles tendon. One week after tenotomy there is a high immunostaining for PCNA (arrows) at the tendon's insertion site (A), at the peri-vascular areas (B) and at the tendon's midsubstance (C) in group A (no NSAID). On the other hand, in group D (NSAID) there is a low immunostaining (D). Bar $=50 \mu \mathrm{m}$.

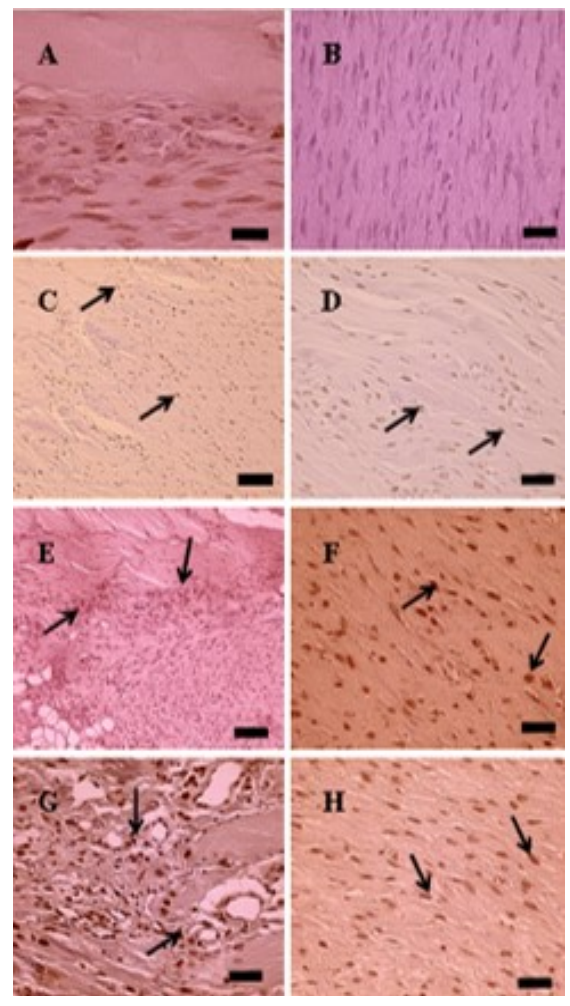

Figure 2. Histology of the longitudinal section of the Achilles tendon. The musculotendinous junction is shown to the left and the mid-subtance of the tendon to the right, 2 weeks after tenotomy. The intensity of the PCNA immunostaining in group A was weak ((A), (B)) but was moderate to high (arrows) in group B ((C), (D)), group C ((E), (F)) and group D ((G), (H)). Bar: $30 \mu \mathrm{m}$ in $A, 50 \mu \mathrm{m}$ in $B, F, G$ and $H, 100 \mu \mathrm{m}$ in $C, D$ and $E$.

junction while in the other 3 groups, the highest marking was found at the tendon's mid-substance, the opposite of what was found at two weeks (Figure 3).

At 8 weeks, no immunostaining was observed. 


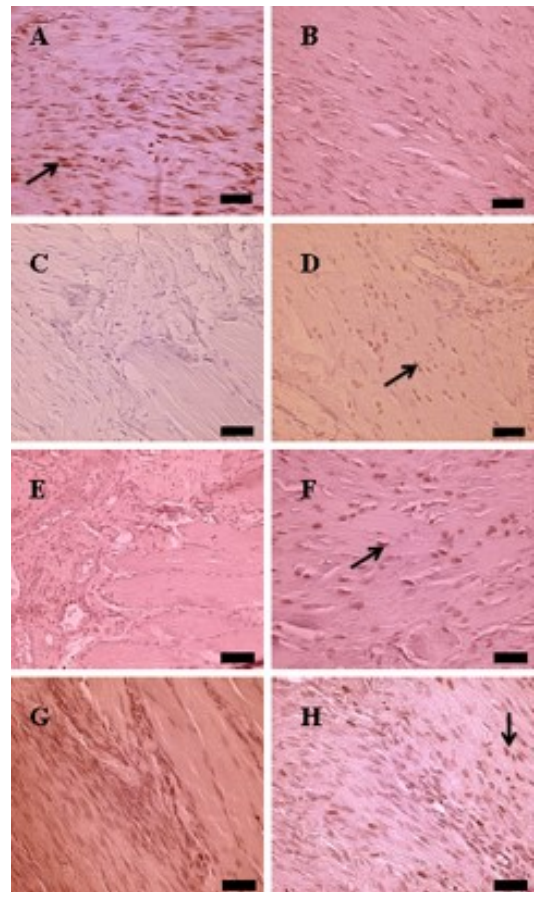

Figure 3. Histology of the longitudinal section of the Achilles tendon. The musculotendinous junction is shown to the left and the mid-subtance of the tendon to the right, 4 weeks after tenotomy. The intensity of the PCNA immunostaining was weak in all groups but was stronger (arrow) in the musculotendinous junction of group $\mathrm{A}$ and in the mid-substance of groups B, C and D. Bar $=100 \mu \mathrm{m}$ in E and $50 \mu \mathrm{m}$ in all others.

\subsection{Biomechanics}

All ruptures occurred at the musculotendinous junction and not at the tenotomy site. The ultimate load values found are shown in Figure 4.

The average ultimate load (and standard deviation) of the animals that were submitted to exercise (groups B and C) was $29.1 \mathrm{~N}$ (5.9) compared to $26.7 \mathrm{~N}$ (6.9) of the animals that did not run (groups A and D) $(P$ value $=0.12)$. The groups that received NSAID (B and D) had an average ultimate load of $28.6 \mathrm{~N}$ (7.1) while the groups that did not receive NSAID (A and C) had an average ultimate load of $27.3 \mathrm{~N}(5.9)(P$ value $=0.40)$.

At two weeks, the average ultimate load (and standard deviation) of the animals that were submitted to exercise (groups B and C) was $28.8 \mathrm{~N}$ (7.0) compared to $29.7 \mathrm{~N}$ (5.6) of the animals that did not run (groups A and D) ( $P$ value $=0.70$ ) (Figure 5). The groups that received NSAID (B and D) had an average ultimate load of $31.5 \mathrm{~N}$ (5.9) while the groups that did not receive NSAID (A and C) had an average ultimate load of $27.0 \mathrm{~N}(6.0)(P$ value $=0.09)$.

At four weeks, the average ultimate load (and standard deviation) of the animals that were submitted to exercise (groups B and C) was $27.7 \mathrm{~N}(5.5)$ compared to $25.8 \mathrm{~N}$ (7.1) of the animals that did not run (groups A and D) ( $P$ value $=0.47$ ) (Figure 5). The groups that received NSAID (B and D) had an average ultimate load of $25.3 \mathrm{~N}$ (6.1) while the groups that did not receive NSAID (A and C) had an average ultimate load of $28.3 \mathrm{~N}(6.3)(P$ value $=0.25)$. 

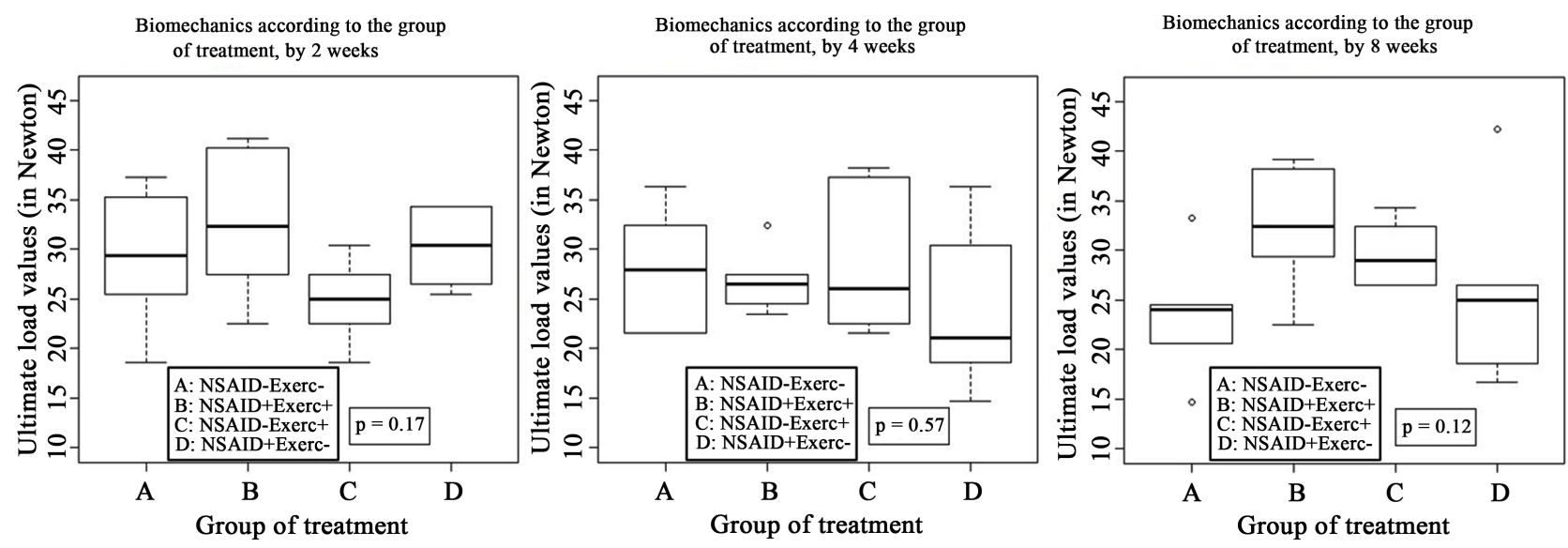

Figure 4. Boxplots charts depicting the analysis of variance of the ultimate load (Newton) of the injured tendon, by weeks after injury.
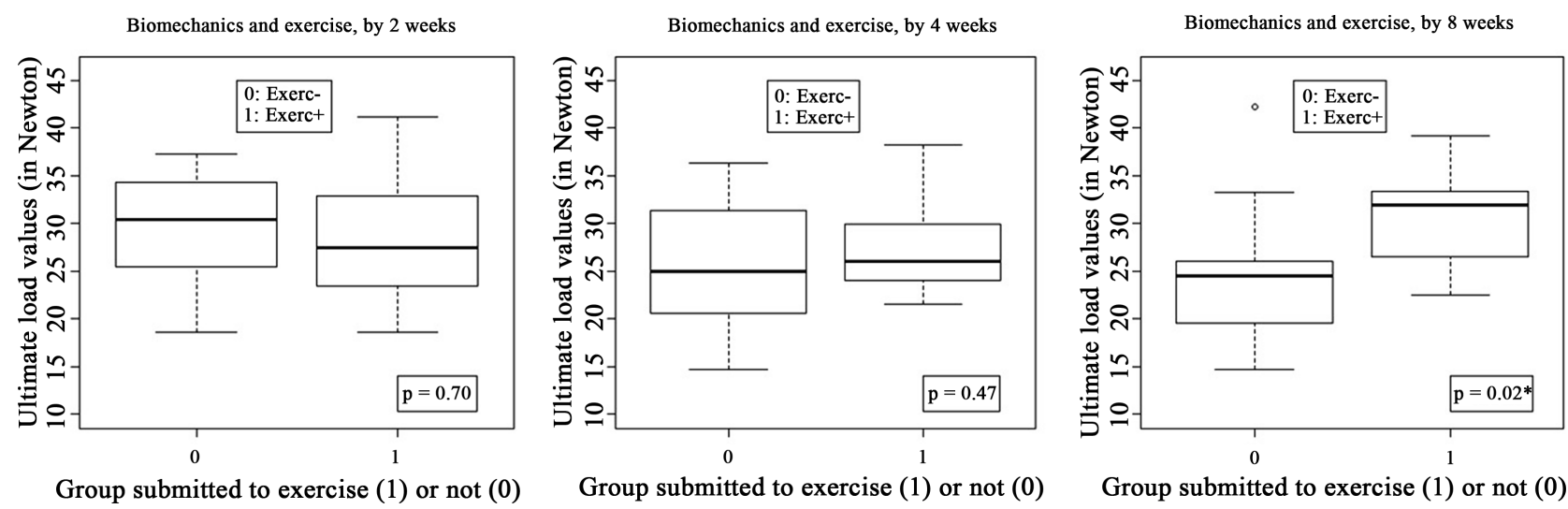

Figure 5. Boxplot graphs depicting the ultimate load values (in Newton) of the injured tendon according to exercise and weeks after injury and adjusted for NSAID use. A significant difference was found at 8 weeks with a higher ultimate load in the exercise group.

At eight weeks, the average ultimate load (and standard deviation) of the animals that were submitted to exercise (groups B and C) was $31.0 \mathrm{~N}(5.0)$ compared to $24.6 \mathrm{~N}$ (7.4) of the animals that did not run (groups A and D) ( $P$ value $=0.02$ ) (Figure 5). The groups that received NSAID (B and D) had an average ultimate load of $29.0 \mathrm{~N}$ (8.1) while the groups that did not receive NSAID (A and C) had an average ultimate load of $26.6 \mathrm{~N}(5.7)(P$ value $=0.35)$.

\subsection{Gait Analysis}

Gait analysis showed a significant difference between animals that received NSAID and animals that did not. Animals that received NSAID presented with a more stable gait cycle with no significant difference between the different time points $(P$ value $=0.94)$.

The animals that did not receive NSAID showed a significant increase in the gait cycle time between the 2 nd and 4 th weeks after the tenotomy ( $P$ value $=$ $0.001)$, confirmed by the Kruskal-Wallis test $(P$ value $=0.006)$. Analysis with the Bonferroni method showed the following differences: between the 2nd and 5th 
weeks $(P$ value $=0.03)$, between the 2 nd and 6 th week $(P$ value $=0.03)$, between the 2 nd and 7 th week $(P$ value $=0.002)$ and between the 4 th and 7 th weeks $(P$ value $=0.03)$ (Figure 6$)$. The other parameters evaluated (time of stance phase, time of swing phase and pace frequency) showed the same pattern (Graphs not shown).

\section{Discussion}

There are numerous advantages in using small animals in an experimental model [23] like the ones used in this study. Nonetheless, the Achilles tendon in these animals is so small that the use of a microscope is of major importance so that accuracy is obtained.

Immobilization, frequently used after a tendon injury and after surgical or conservative treatments, can alter the regeneration process [24]. In spite of this, the role of exercise in the inflammatory, proliferative and remodeling phases of tendon regeneration is still not clear and the ideal rehabilitation protocol that would provide functional gain and no tissue injury is still unknown [25].

In our study, we observed that 4 weeks after tenotomy there was a decline in the ultimate load except for group $C$ that was only submitted to exercise (this group had the smallest ultimate load at 2 weeks). Also, animals submitted to exercise or to the use of NSAID had an improvement in the ultimate load between the 4th and 8th weeks; this increase was more obvious in the groups submitted to the isolated intervention of exercise or NSAID and it was maintained after adjusting for time, exercise and the use of NSAID.

There is a specificity for the role of exercise in the benefits for tendon healing. The tendons have a good response to aerobic exercise like swimming and running but not to strengthening exercises [26]. It is also known that running has better effects on tendon healing than swimming [27]. This study has confirmed
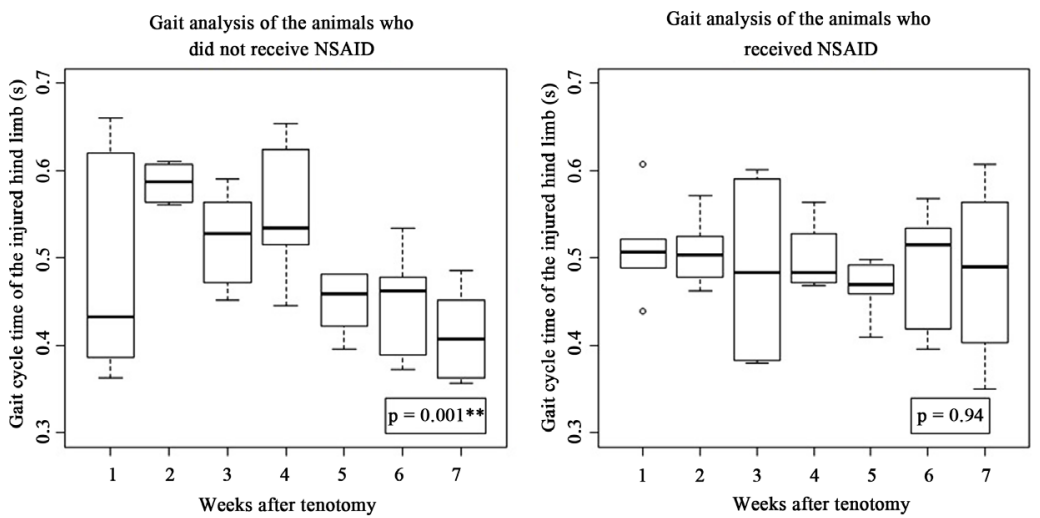

Figure 6. Boxplots showing the gait cycle of the injured hind limb, divided by the use or not of NSAID and by weeks after tenotomy. The animals that did not receive NSAID showed a significant increase in the gait cycle confirmed by the Kruskal-Wallis test $(P$ value $=0.006)$. Analysis with the Bonferroni method showed the following differences: between the 2 nd and 5 th weeks $(P$ value $=0.03$ ), between the 2 nd and 6 th week $(P$ value $=$ $0.03)$, between the 2 nd and 7 th week $(P$ value $=0.002)$ and between the 4 th and 7 th weeks $(P$ value $=0.03)$ 
that aerobic exercise, with a gradual increase in intensity and started one week after a total Achilles tendon rupture, has a positive effect on the biomechanical characteristics of the tendon showed by an improvement over time of the tendon's ultimate load. Other studies confirm this as they have demonstrated that exercise benefits tendon regeneration when exercise is initiated early after the injury but not immediately after [28] [29] [30] and Nilsson-Helander et al. [31] have shown that early tendon mobilization is the most important aspect in the Achilles tendon rupture treatment. Our study also suggests that exercise has better effects on tendon healing when the use of NSAID is initiated before and, if this can be confirmed by human clinical trials, it could have an important impact on tendon treatment.

Previous studies have shown that the use of NSAID in murine tendon injuries can lead to structural changes like the reduction of the edema, of the number of inflammatory cells and of collagen expression, organization and maturation changes, but with no modification in biomechanical or functional tendon characteristics [32]. At 4 and 8 weeks, we did not see a significant difference in ultimate loads between the groups that received NSAID and the groups that did not although we did see a trend towards significance $(P$ value $=0.09)$ at two weeks with the group that received NSAID with a higher ultimate load.

When gait analysis was assessed, the animals that received NSAID showed better stability with regards to the parameters evaluated suggesting benefits in the drug use. With this, we could speculate a possible protection against a re-rupture that is the most unwanted adverse effect after a previous tendon injury [33], but this needs to be better investigated.

Cell proliferation is an important fact after tendon injury and is fundamental in the healing process [12]. Normal tendon does not express PCNA but after injury it can be seen after 3 days and it has an important role in DNA repair after tissue injury [34]. According to Galatz et al. [35] the height of cellular proliferation after injury occurs at day 7 and decreases at 4 weeks, result similar to our group A, while $\mathrm{Wu}$ YF et al. [36] found that most of the proliferation occurs between weeks 2 and 3 after injury, a similar result found in the remaining groups of this study that were submitted to exercise or to the use of NSAID. The use of these interventions, exercise or NSAID, seem to delay the stimulus for cell proliferation but the consequence of this delay must the better evaluated in future studies.

We also observed that there was a difference in the site of cell proliferation over time. The progressive PCNA expression at different sites of the injured tendon illustrates the need to study this dynamic process over time with special attention to the musculotendinous junction. Other studies have also shown the different patterns of PCNA expression. Kawamura et al. [37], for instance, found proliferating cells first seen in the tendon-bone interface, with a gradual progression into the tendon, whereas Lui et al. [38] demonstrated higher cell density in the center of the wound at day 4 , but at the periphery of the wound at day 28 . 
Future studies are needed to better investigate what cell is proliferating and where these cells originate.

Dimmen et al. [11] demonstrated that all tendon ruptures occurred at the injury site during the biomechanical testing and many previous studies lack the information of where the tendon ruptures. In our study, the vast majority of the ruptures occurred at the musculotendinous junction and the reason for this must be better investigated. An important limitation of this study, and that could explain this rupture site, is that these animals did not have a chronic tendinopathy and because of this did not have a higher probability of re-rupture or greater tendon fragility at the tenotomy site.

Future studies are needed to appraise results after using selective NSAID that could be more effective in reducing the inflammatory response after a tendon injury and to evaluate the collagen fibers and the expression of specific factors like TGF- $\beta$ and VEGF in the tendon regeneration process.

\section{Conclusion}

Aerobic exercise, initiated early after tendon injury, is beneficial for the biomechanical aspects that occur during tendon regeneration. The combined use of NSAID provided better gait stability and this could protect against tendon re-rupture.

\section{Acknowledgements}

The authors wish to thank: Sheila Ingham for the help with the English language, Thiago Gagliano for the help with the article's structure, the employees at the Mechanical Engineering Lab at the UERJ (Manuel Augusto, Carlos Alberto Ferreira and mainly Wilson Lourenço) for the support with the biomechanical testing, Cleber Christianes Souza da Silva from the Zoology Lab at the UERJ for the help with the filming of the animals and data interpretation, Ana Lúcia Rosa Nascimento for the help with the histological analysis, Nádia Cristina Pinheiro Rodrigues for the help with the statistical analysis and finally Cláudio Sérgio Corrêa Lau and Hamilton Jackson Brito de Oliveira for the animal's care.

This study has received financial support from Fundação de Amparo à Pesquisa do Estado do Rio de Janeiro-Faperj through scholarships to Clara Morena Abreu Raposo (2009 to 2011) and from grants to Liszt Palmeira de Oliveira.

\section{Conflicts of Interest}

The authors declare no conflicts of interest regarding the publication of this paper.

\section{References}

[1] Chen, T.M., Rozen, W.M., Pan, W.R., Ashton, M.W., Richardson, M.D. and Taylor, G.I. (2009) The Arterial Anatomy of the Achilles Tendon: Anatomical Study and 
Clinical Implications. Clinical Anatomy, 22, 377-385. https://doi.org/10.1002/ca.20758

[2] Spennacchio, P., Vascellari, A., Cucchi, D., Canata, G. and Randelli, P. (2016) Outcome Evaluation after Achilles Tendon Ruptures. A Review of the Literature. Joints, 4, 52-61.

[3] Thompson, J. and Baravarian, B. (2011) Acute and Chronic Achilles Tendon Ruptures in Athletes. Clinics in Podiatric Medicine and Surgery, 28, 117-135. https://doi.org/10.1016/j.cpm.2010.10.002

[4] Costa, M.T. and Hungria Neto, J.S. (2007) Estudo comparativo dos métodos conservador e cirúrgico para tratamento das lesões agudas do tendão do calcâneo. Acta Ortopédica Brasileira, 15, 50-54. https://doi.org/10.1590/S1413-78522007000100011

[5] Khan, R.J. and Carey Smith, R.L. (2010) Surgical Interventions for Treating Acute Achilles Tendon Ruptures. Cochrane Database of Systematic Reviews, No. 9, CD003674. https://doi.org/10.1002/14651858.CD003674.pub4

[6] Tan, G., Sabb, B. and Kadakia, A.R. (2009) Non-Surgical Management of Achilles Ruptures. Foot and Ankle Clinics, 14, 675-684. https://doi.org/10.1016/j.fcl.2009.08.004

[7] Suchak, A.A., Bostick, G.P., Beaupré, L.A., Durand, D.C. and Jomha, N.M. (2008) The Influence of Early Weight-Bearing Compared with Non-Weight-Bearing after Surgical Repair of the Achilles Tendon. The Journal of Bone and Joint Surgery, 90, 1876-1883. https://doi.org/10.2106/JBJS.G.01242

[8] Willits, K., Amendola, A., Bryant, D., Mohtadi, N.G., Giffin, J.R., Fowler, P., et al. (2010) Operative versus Nonoperative Treatment of Acute Achilles Tendon Ruptures: A Multicenter Randomized Trial Using Accelerated Functional Rehabilitation. The Journal of Bone \& Joint Surgery, 92, 2767-2775. https://doi.org/10.2106/JBJS.I.01401

[9] Yotsumoto, T., Miyamoto, W. and Uchio, Y. (2010) Novel Approach to Repair of Acute Achilles Tendon Rupture: Early Recovery without Postoperative Fixation or Orthosis. The American Journal of Sports Medicine, 38, 287-292. https://doi.org/10.1177/0363546509351557

[10] Langberg, H., Boushel, R., Skovgaard, D., Risum, N. and Kjaer, M. (2003) Cyclo-Oxygenase-2 Mediated Prostaglandin Release Regulates Blood Flow in Connective Tissue during Mechanical Loading in Humans. The Journal of Physiology, 551, 683-689. https://doi.org/10.1113/jphysiol.2003.046094

[11] Dimmen, S., Engebretsen, L., Nordsletten, L. and Madsen, J.E. (2009) Negative Effects of Parecoxib and Indomethacin on Tendon Healing: An Experimental Study in rats. Knee Surgery, Sports Traumatology, Arthroscopy, 17, 835-839.

https://doi.org/10.1007/s00167-009-0763-7

[12] Tsai, W.-C., Hsu, C.-C., Chou, S.-W., Chung, C.-Y., Chen, J. and Pang, J.-H.S. (2007) Effects of Celecoxib on Migration, Proliferation and Collagen Expression of Tendon Cells. Connective Tissue Research, 48, 46-51.

https://doi.org/10.1080/03008200601071295

[13] Virchenko, O., Skoglund, B. and Aspenberg, P. (2004) Parecoxib Impairs Early Tendon Repair but Improves Later Remodeling. The American Journal of Sports Medicine, 32, 1743-1747. https://doi.org/10.1177/0363546504263403

[14] Chiodo, C.P., Glazebrook, M., Bluman, E.M., Cohen, B.E., Femino, J.E., Giza, E., et al. (2010) Diagnosis and Treatment of Acute Achilles Tendon Rupture. Journal of the American Academy of Orthopaedic Surgeons, 18, 503-510. 
https://doi.org/10.5435/00124635-201008000-00007

[15] Twaddle, B.C. and Poon, P. (2007) Early Motion for Achilles Tendon Ruptures: Is Surgery Important? A Randomized, Prospective Study. The American Journal of Sports Medicine, 35, 2033-2038. https://doi.org/10.1177/0363546507307503

[16] Ulreich, N., Kainberger, F., Huber, W. and Nehrer, S. (2002) Achilles Tendon and Sports. Radiology, 42, 811-817. https://doi.org/10.1007/s00117-002-0800-8

[17] Huang, T.-F., Perry, S.M. and Soslowsky, L.J. (2004) The Effect of Overuse Activity on Achilles Tendon in an Animal Model: A Biomechanical Study. Annals of Biomedical Engineering, 32, 336-341. https://doi.org/10.1023/B:ABME.0000017537.26426.76

[18] Kurtz, R.C., Almeida, A.L., Apfel, M.R., Elias, N. and Mesquita, K.C. (1996) Cicatrização nas lesões agudas do tendão calcaneano. Revista Brasileira de Ortopedia, 31, 857-861.

[19] Christensen, B., Dandanell, S., Kjaer, M. and Langberg, H. (2011) Effect of Anti-Inflammatory Medication on the Running-Induced Rise in Patella Tendon Collagen Synthesis in Humans. Journal of Applied Physiology, 110, 137-141. https://doi.org/10.1152/japplphysiol.00942.2010

[20] Gomes Barbato, K.B., de Almeida, G., da Costa, J., Rodriguez, L., Raposo, C., Dias, H., et al. (2018) Complete Achilles Tenotomy: A New Improved Experimental Surgical Technique in Rats. Open Journal of Animal Sciences, 8, 1-11. https://doi.org/10.4236/ojas.2018.81001

[21] Giordano, V., Giordano, M., Knackfuss, I.G., Apfel, M.I.R. and Gomes, R.D.C. (2003) Effect of Tenoxicam on Fracture Healing in Rat Tibiae. Injury, 34, 85-94. https://doi.org/10.1016/S0020-1383(02)00199-7

[22] Véras-Silva, A.S., Mattos, K.C., Gava, N.S., Brum, P.C., Negrão, C.E. and Krieger, E.M. (1997) Low-Intensity Exercise Training Decreases Cardiac Output and Hypertension in Spontaneously Hypertensive Rats. American Journal of Physiology, 273, H2627-H2631.

[23] Warden, S.J. (2007) Animal Models for the Study of Tendinopathy. British Journal of Sports Medicine, 41, 232-240. https://doi.org/10.1136/bjsm.2006.032342

[24] Bring, D.K.-I., Reno, C., Renstrom, P., Salo, P., Hart, D.A. and Ackermann, P.W. (2009) Joint Immobilization Reduces the Expression of Sensory Neuropeptide Receptors and Impairs Healing after Tendon Rupture in a Rat Model. Journal of Orthopaedic Research, 27, 274-280. https://doi.org/10.1002/jor.20657

[25] Fouda, M.B., Thankam, F.G., Dilisio, M.F. and Agrawal, D.K. (2017) Alterations in Tendon Microenvironment in Response to Mechanical Load: Potential Molecular Targets for Treatment Strategies. American Journal of Translational Research, 9, 4341-4360.

[26] Buchanan, C.I. and Marsh, R.L. (2001) Effects of Long-Term Exercise on the Biomechanical Properties of the Achilles Tendon of Guinea Fowl. Journal of Applied Physiology, 90, 164-171. https://doi.org/10.1152/jappl.2001.90.1.164

[27] See, E.K.N., Ng, G.Y.F., Ng, C.O.Y. and Fung, D.T.C. (2004) Running Exercises Improve the Strength of a Partially Ruptured Achilles Tendon. British Journal of Sports Medicine, 38, 597-600. https://doi.org/10.1136/bjsm.2003.007120

[28] Enwemeka, C.S., Maxwell, L.C. and Fernandes, G. (1992) Ultrastructural Morphometry of Matrical Changes Induced by Exercise and Food Restriction in the Rat Calcaneal Tendon. Tissue Cell, 24, 499-510.

https://doi.org/10.1016/0040-8166(92)90066-G 
[29] Freedman, B.R., Gordon, J.A., Bhatt, P.R., Pardes, A.M., Thomas, S.J., Sarver, J.J., et al. (2016) Nonsurgical Treatment and Early Return to Activity Leads to Improved Achilles Tendon Fatigue Mechanics and Functional Outcomes during Early Healing in an Animal Model: Effect of Repair and Return to Activity on Achilles Tendon Healing. Journal of Orthopaedic Research, 34, 2172-2180. https://doi.org/10.1002/jor.23253

[30] Godbout, C., Ang, O. and Frenette, J. (2006) Early Voluntary Exercise Does Not Promote Healing in a Rat Model of Achilles Tendon Injury. Journal of Applied Physiology, 101, 1720-1726. https://doi.org/10.1152/japplphysiol.00301.2006

[31] Nilsson-Helander, K., Silbernagel, K.G., Thomeé, R., Faxén, E., Olsson, N., Eriksson, B.I., et al. (2010) Acute Achilles Tendon Rupture: A Randomized, Controlled Study Comparing Surgical and Nonsurgical Treatments Using Validated Outcome Measures. The American Journal of Sports Medicine, 38, 2186-2193. https://doi.org/10.1177/0363546510376052

[32] Marsolais, D., Côté, C.H. and Frenette, J. (2003) Nonsteroidal Anti-Inflammatory Drug Reduces Neutrophil and Macrophage Accumulation But Does Not Improve Tendon Regeneration. Laboratory Investigation, 83, 991-999. https://doi.org/10.1097/01.LAB.0000078688.07696.AC

[33] Metz, R., van der Heijden, G.J.M.G., Verleisdonk, E.-J.M.M., Kolfschoten, N., Verhofstad, M.H.J. and van der Werken, C. (2011) Effect of Complications after Minimally Invasive Surgical Repair of Acute Achilles Tendon Ruptures: Report on 211 Cases. The American Journal of Sports Medicine, 39, 820-824. https://doi.org/10.1177/0363546510392012

[34] Maga, G. and Hubscher, U. (2003) Proliferating Cell Nuclear Antigen PCNA, a Dancer with Many Partners. Journal of Cell Science, 116, 3051-3060. https://doi.org/10.1242/jcs.00653

[35] Galatz, L.M., Sandell, L.J., Rothermich, S.Y., Das, R., Mastny, A., Havlioglu, N., et al. (2006) Characteristics of the Rat Supraspinatus Tendon during Tendon-to-Bone Healing after Acute Injury. Journal of Orthopaedic Research, 24, 541-550. https://doi.org/10.1002/jor.20067

[36] Wu, Y.F., Chen, C.H., Cao, Y., Avanessian, B., Wang, X.T. and Tang, J.B. (2010) Molecular Events of Cellular Apoptosis and Proliferation in the Early Tendon Healing Period. Journal of Hand Surgery, 35, 2-10.

https://doi.org/10.1016/j.jhsa.2009.10.021

[37] Kawamura, S., Ying, L., Kim, H.-J., Dynybil, C. and Rodeo, S.A. (2005) Macrophages Accumulate in the Early Phase of Tendon-Bone Healing. Journal of Orthopaedic Research, 23, 1425-1432. https://doi.org/10.1016/j.orthres.2005.01.014

[38] Lui, P.P.Y., Cheuk, Y.C., Hung, L.K., Fu, S.C. and Chan, K.M. (2007) Increased Apoptosis at the Late Stage of Tendon Healing. Wound Repair and Regeneration, 15, 702-707. https://doi.org/10.1111/j.1524-475X.2007.00276.x 